\title{
Geostatistics/reliability based risk analysis of the Vajont landslide
}

\author{
M. Guarascio, M. Lombardi, G. Rossi \& G. Sciarra \\ Safety Engineering, University of Rome "La Sapienza", Italy
}

\begin{abstract}
This paper is devoted to the probabilistic analysis of the Vajont landslide event according to the basic statements of risk analysis. In particular, the goal is to find out a regressive estimate of the landslide rate of movement in terms of the rainfall, $R_{f}$, and the reservoir level, $R_{l}$, time evolution during the test period. This will be obtained by developing a kind of geostatistical analysis on the space of standardized variables $\left(R_{f}, R_{l}\right)$, based on classical ordinary kriging. Risk analysis will be formulated introducing a suitable safety margin function, depending on the mass of the slide, $M$, on its initial velocity, $v_{i n}$, and the amplitude, $H$, of the water wave flowing out of the Vajont basin.
\end{abstract}

Keywords: geostatistics, reliability, risk analysis.

\section{Introduction}

The Vajont landslide of 9th October 1963 was one of the major disasters due to the occurrence of a landslide in the recent past. A landslide of $26010^{6} \mathrm{~m}^{3}$ of materials detached from Mt. Toc, in the north of Italy, falling into the Vajont hydro-power basin and consequently inducing a wave on the opposite side of the lake, the crest of which was about $200 \mathrm{~m}$ higher than the level of the concrete dam. Water coming back into the reservoir, which at that time had been filled up to the level of $700 \mathrm{~m}$, caused about $5010^{6} \mathrm{~m}^{3}$ of water to move out of the lake plunging onto Longarone, Fig. 1, which was completely destroyed, and the suburbs of Rivalta, Pirago, Faè, Villanova and Codissago.

Hendron and Patton [1] proved that it was both the occurrence of a significant rainfall level, in the days before the disaster, and the high level of the reservoir to trigger the landslide. In particular, the phenomenon which was mainly responsible for triggering the catastrophic event was the presence, under an ancient landslide, of a stratum of impermeable clays which forced the pore pressure inside and below 


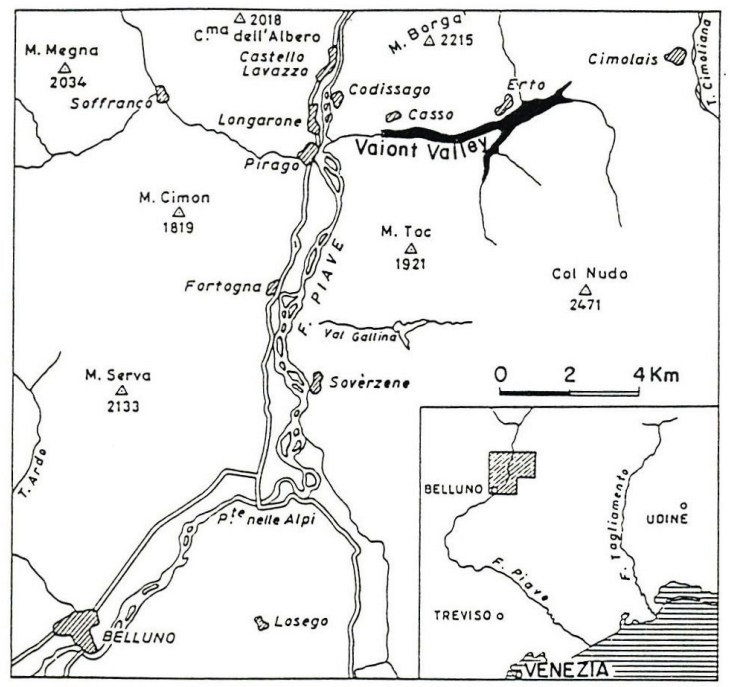

(after Selli and Trevisan, 1964)

Figure 1: Map of the Vajont site.

the slide mass to increase up to the limit state, when it induced the clays to abruptly evaporate and consequently the shear strength to vanish. We refer to [1], for a detailed discussion of the slide mechanism. In the following figures the rainfall time evolution, the increase of the reservoir level and the variation of the slide rate of movement, from January 1960 to 9th October 1963, Figs. 2 and 3, are drawn. In Fig. 4 the time evolution of the rainfall, averaged over 7, 15 and 30 days supports, is also depicted in order to account for the effect of groundwater accumulation and increase of the pore-water pressure.

On the basis of the data relative to the rainfall, the reservoir level and the slide rate of movement up to the early days of September 1963 we aim to find out a suitably defined regression procedure which allows for the extrapolation of the critical values of the sliding rate, associated with the effective conditions which characterized the state of the basin and the dam on 9th October 1963.

The adopted procedure is based on geostatistics and, in particular, on a suitable regression procedure, in which the covariance among the values of the landslide rate of movement is estimated via proper dissimilarity function. The following section is devoted to present the main features of the method.

\section{Geostatistical analysis of the landslide rate of movement}

Geostatistics is generally used for modeling the behavior of spatially distributed, partially unknown, fields: starting from the knowledge of the values of the field in 


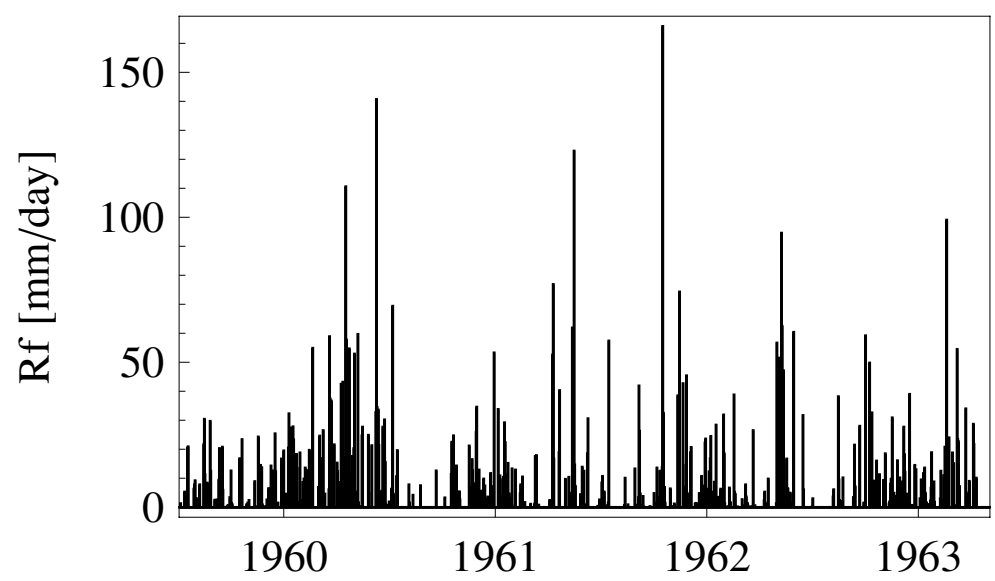

Figure 2: Daily rainfall data from Erto metereological station.
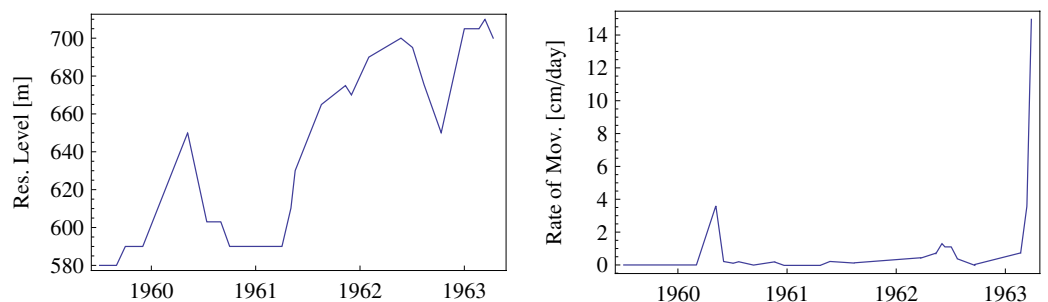

Figure 3: Averaged rainfalls over a period of 7, 15 or 30 days before the current date.
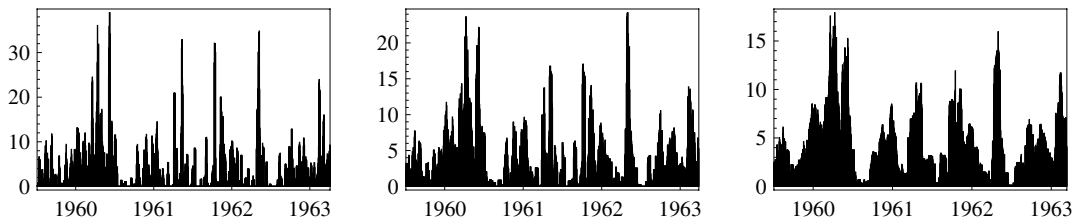

Figure 4: Time variation of the reservoir level and the slide rate of movement.

suitable sample points, of a given domain, the goal is that of estimating, via a kind of regression procedure, the values of the field in the missing points, i.e. in those points of the domain where measures are not available. In particular, monovariate geostatistics regards the value of the unknown field in every point of the domain as a random variable, which in other words means regarding the field as a random 
function of space. The covariance among the random variables which describes the value of a random field $f$ in different points of the domain is prescribed, in the framework of stationary processes, in terms of a suitable dissimilarity measure: if

$$
\gamma(h)=\frac{1}{2} E\left[(f(X+h)-f(x))^{2}\right]
$$

is the variogram associated to the stationary random field $f(E[\cdot]$ indicates the expected value operator.) which depends only on the difference of positions $h$, then the corresponding covariance function is given by

$$
C(h)=C(0)-\gamma(h)
$$

Starting from the knowledge of the covariance function, estimating the value of $f$ in a suitable test point $x_{0}$ is a straightforward application of standard regression technique. In the framework of geostatistics this is labeled as kriging (see e.g. [2]). In particular within the framework of ordinary kriging the value of $f\left(x_{0}\right)$ is provided by the following linear combination of the values of the random field $f$ in the available sample points $x_{i}$ :

$$
f\left(x_{0}\right)=\sum_{i=1}^{n} a_{i} f\left(x_{i}\right)
$$

here the weights $a_{i}$, which are going to be determined via linear regression, are constrained to sum up to 1 .

In our context we enlarge the concept of physical domain to the case of the $2 D$ modeling space $\mathcal{P}_{\left(R_{f}^{\prime}, R_{l}^{\prime}\right)}$ given by the cartesian product of the standardized rainfall times the standardized reservoir level.

$$
R_{f}^{\prime}=\frac{R_{f}-\mu_{R_{f}}}{\sigma_{R_{f}}} \quad R_{l}^{\prime}=\frac{R_{l}-\mu_{R_{l}}}{\sigma_{R_{l}}}
$$

Bearing in mind the common dependence of rainfall, reservoir level and landslide rate of movement on time, one can introduce a suitable dissimilarity measure $\gamma_{R M}$ among different values of the random field which describes the rate of movement of the landslide, for different points in the $\mathcal{P}_{\left(R_{f}^{\prime}, R_{l}^{\prime}\right)}$ space. In Fig. 5 the localization of the sample points in the considered modeling space are drawn; the distinction among the three different picture resides in the time interval over which both the rainfall and the reservoir level are averaged; in particular the time supports of 7 , 15 and 30 days have been considered.

The variogram estimation, based on the available data (see e.g. Fig. 5, for the case of 30-days support) in the modeling space, provides the basic instrument for extrapolating the value of the landslide rate of movement, relative to the rainfall and reservoir level which were effectively measured when the collapse conditions showed up. 

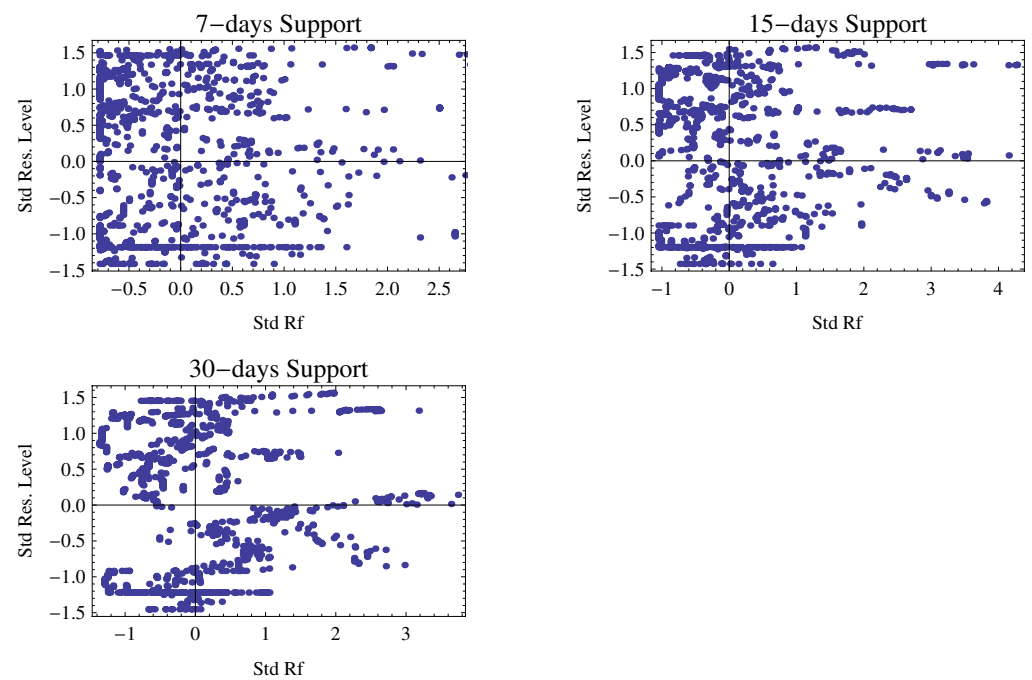

Figure 5: Sample point spatial distribution in the domain of standardized rainfall and reservoir level.

The experimental variogram is given by the classical Matheron estimator (see [3]):

$$
\gamma(h)=\frac{1}{2 n_{c}} \sum_{i=1}^{n_{c}}\left[f\left(x_{i}+h\right)-f\left(x_{i}\right)\right]^{2}
$$

where $n_{c}$ is the number of points in the modeling space having a relative distance equal to $h \pm \Delta h / 2, \Delta h$ being the wideness of each class. We remark that in this context $h$ measures the distance between distinct points in the space of standardized rainfalls and reservoir levels. Every sample point is associated to a given time (a day in the range 1st January 1960-9th October 1963) which implies the considered distance to account for time dissimilarity.

\section{Kriging the landslide rate of movement}

Geostatistical ordinary kriging is used in order to develop smooth maps of the sliding rate of movement. The dissimilarity between distinct values of this field, defined over the modeling space $\mathcal{P}_{\left(R_{f}^{\prime}, R_{l}^{\prime}\right)}$, provides as an estimate of the covariance between values of the random field associated (in the sense we have previously made precise) to distinct points of the sample domain; kriging provides a regression estimate of the sliding rate of movement in those point where direct measures are not available. In particular it allows for determining a time extrapolation of what happened on 9th October 1963, starting from data spanning the time life of the dam up the early days of September 1963. Different estimates of the 


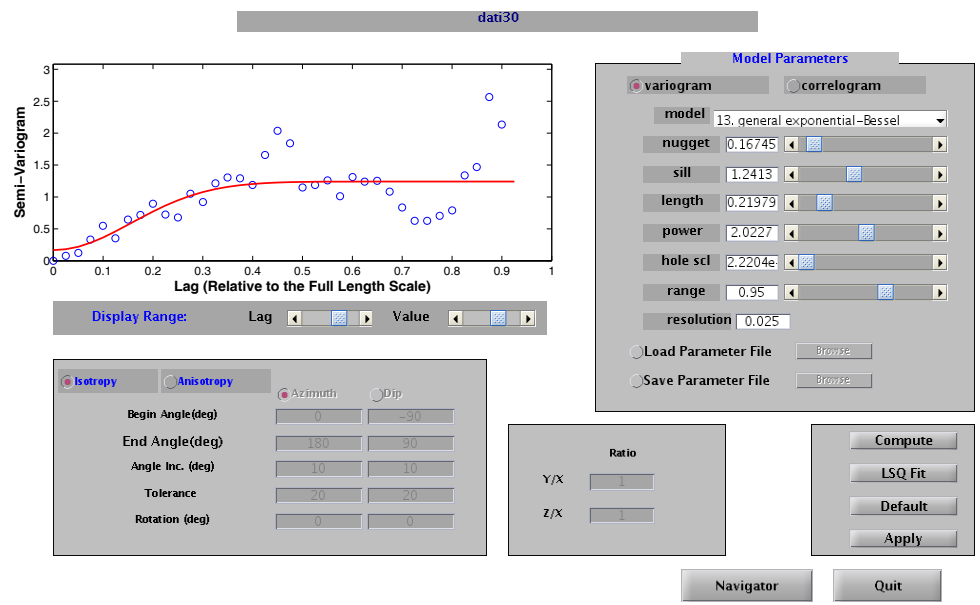

Figure 6: Example of variogram estimation in the framework of stationary processes. The worksheet is an output of the easykrig tool running under matlab.
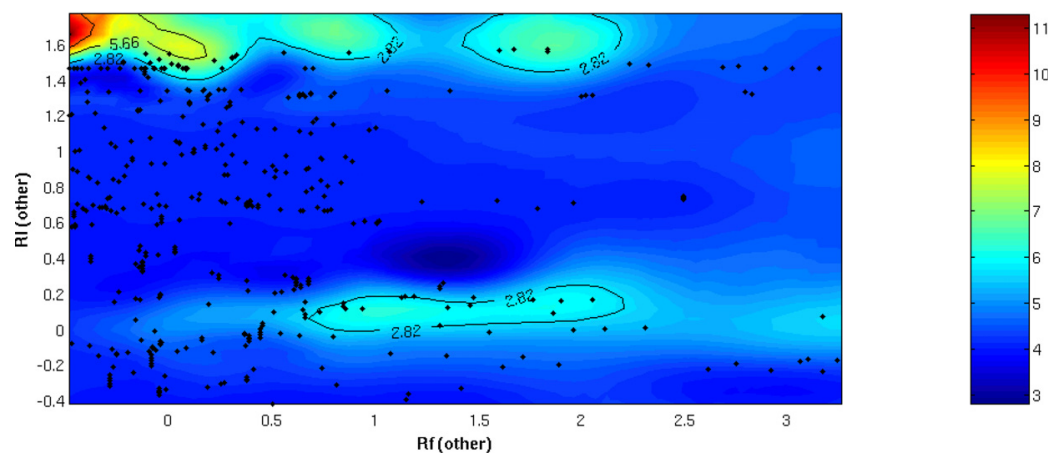

Figure 7: Ordinary Kriging map, for data averaged on a 7 days support.

slide rate of movement are drawn in Figs. 7-9, according to the different supports used for computing both average rainfalls and reservoir levels.

The map relative to 30 days support data allows for identifying three peaks centered in the following points of the space $\mathcal{P}_{\left(R_{f}^{\prime}, R_{l}^{\prime}\right)}$ :

$$
P_{1} \equiv(3,0.4), \quad P_{2} \equiv(1.6,1.6), \quad P_{3} \equiv(0.5,1.6),
$$

which correspond to the landslide of November 1960, to the state of the dam-basin system a month before the critical event and to the critical event itself, respec- 

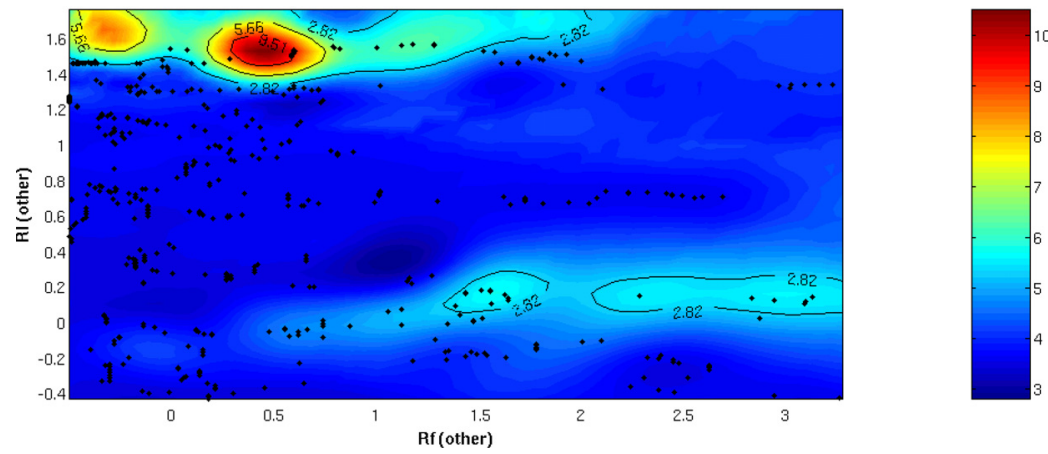

Figure 8: Ordinary Kriging map, for data averaged on a 15 days support.
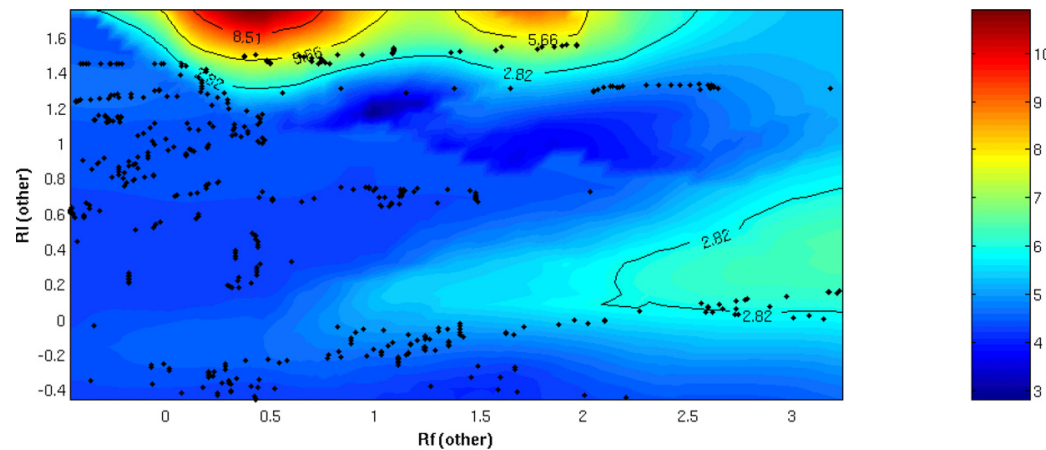

Figure 9: Ordinary Kriging map, for data averaged on a 30 days support.

tively. This kind of behavior can be also recovered in the maps relative to 7 and 15 days support data, even if a not so evident identification of the critical dates is in these cases available. Apparently the estimated value of the landslide rate of movement are consistent with the showing up of the critical conditions in the early days of October 1963, even if not a real jump in the velocity of the slide can be extrapolated. This is obviously due to the regularizing effect produced by regression (kriging). However it is clear that an estimated landslide rate of movement varying between 7 and $12 \mathrm{~cm} /$ day can not be regarded as physiological, especially in case of complex geomorphological/geographical sites as the Vajont valley was. According with these remark it is worth to notice that it was neither the instantaneous rainfall nor the corresponding reservoir level to affect the strength of the Mt. Toc ancient landslide, but conversely the effect of pore fluid segregation inside and below the slide mass, which can be measured and accounted for considering the average rainfall and reservoir level for a 30 days monitoring time-window. 


\section{A reliability based risk analysis procedure}

Starting from the estimate of the landslide rate of movement a risk analysis could be developed, accounting for the following control parameters:

- the velocity $\left(v_{i}\right)$ of the water mass which passed above the crest of the dam; this velocity depends on the detaching landslide rate of movement and determines the "initial"kinetic energy of water,

- the amplitude $(H)$ of the wave, with respect to the crest of the dam; this amplitude also depends on the detaching landslide rate of movement but determines the "initial"potential energy of water,

- the mass $(M)$ of the water itself, which is related to the spatial distribution of the landslide rate of movement.

These three parameters determine the total (kinetic) energy of the water mass when reaching the Vajont valley, and in particular the small town of Longarone. In order to develop a probabilistic risk analysis procedure we aim to compare the random variable $\varepsilon$, which describes the kinetic energy of the water and can be regarded as a function of the aforementioned control random parameters, with the energy threshold $\varepsilon_{T}$, which will be regarded as a discrete random variable.

Introducing a safety margin function defined as follows:

$$
M S\left(v_{\text {in }}, H, M\right)=\varepsilon_{T}-\left(\frac{1}{2} M v_{\text {flow }}^{2}+M g H\right)
$$

a proper characterization of the probability of being this safety margin smaller than zero can be obtained via classical arguments of reliability analysis (see e.g. [4] or [5]), starting from the knowledge of the random properties of the control parameters. Apparently the results of the analysis can be validated considering the effects, in term of victims, of the worst critical scenario associated to the effective amount of water which, moving out of the basin, destroyed Longarone. Assuming $\varepsilon_{T}$ to range in a suitable interval, with a prescribed (discrete) probability distribution function, explicit computations can be developed in order to get an estimate of the probability of the safety margin to be negative. The results of the analysis could be summarized by means of an Event Tree diagram, where the non-trivial consequence events are listed together with the corresponding probabilities. An $a d$ hoc measure of damage, consistent with the data of the catastrophic Vajont slide, should be introduced. The result of the procedure will be, therefore presented via suitable $F-N$ curves to be compared with those ones associated to critical natural events, for instance earthquake scenarios.

\section{Conclusions}

In this paper a preliminary analysis of the Vajont landslide, which occurred on 9th October 1963, has been presented. Adopting the approach typical of geostatistics and risk analysis we accomplished the average rainfall and reservoir level in the 30 days before the catastrophic event to be responsible for the landslide (see fig. 9). Several aspects concerning the quantitative estimation of risk measured in 
terms of expected fatalities as well as the comparison of it with suitably identified acceptability criteria need more deepening. Further developments will be devoted to implementing more refined geostatistical methods in order to improve the estimate of the landslide rate of movement; moreover a complete reliability analysis, based on the available data for the control parameters, will be also developed.

\section{References}

[1] Hendron, J. \& Patton, F.D., The vajont slide, a geotechnical analysis based on new geologic observation of the failure surface. Technical report, US Army Corps of engineers - Washington DC, 1985.

[2] Wackernagel, H., Multivariate geostatistics. Springer-Verlag: Southampton and Boston, 1995.

[3] Matheron, G., La Theorie des Variables Regionalisees et ses Applications. Masson Paris, 1965.

[4] Ang, A.S. \& Tang, W.H., Probability Concepts in Engineering Planning and Design Vol. II. Wiley, 1990.

[5] Ditlevsen, O. \& Madsen, H., Structural Reliability Methods. Wiley, 2005. 